\title{
Observed Cosmological Reexpansion in Minimal QFT with Bose and Fermi Fields
}

\author{
Christine Gruber ${ }^{1}$ and Hagen Kleinert ${ }^{1,2}$ \\ ${ }^{1}$ Institut für Theoretische Physik, Freie Universität Berlin, Arnimallee 14, D-14195 Berlin, Germany; \\ ${ }^{2}$ ICRANet, Piazza della Repubblica 10, 65122 Pescara, Italy.
}

(Dated: November 5, 2018)

\begin{abstract}
In this work we aim at explaining the re-acceleration of the expansion of the universe, or equivalently, the hierarchy problem, with the help of a simple field-theoretical model. In particular, we want to account for the notorious discrepancy between the observed value of the cosmological expansion term and theoretical values of the vacuum energy of free quantum fields. Rendered infinite by short-wavelength fluctuations, evaluation up to a cutoff in momentum space at the Planck scale leads to contributions of the order of $10^{76} \mathrm{GeV}^{4}$. The observed value of the cosmic expansion being of the order of $10^{-47} \mathrm{GeV}^{4}$, this is a difference of 123 orders of magnitude. We propose a possible resolution of the hierarchy problem by a cancelation of divergences by equivalent contributions of bosonic and fermionic fields of the system, albeit after some fine-tuning of the parameters of the field theory. We show that in principle nothing beyond conventional ingredients of quantum field theory is necessary to provide us with a possible explanation of the observed dark energy, and thus with a solution to the hierarchy problem.
\end{abstract}

PACS numbers: 95.36.+x, 98.80.Es, 03.70.+k

\section{INTRODUCTION}

Some years ago, astrophysical observations [1] have suggested that the universe is currently in a transition from a matter-dominated state into a phase of accelerating expansion. The cause for this phenomenon has been subject to many speculations, reaching from the modification of geometry [2-4] , over the introduction of a cosmological constant $\Lambda$ to the postulation of new species of quantum fields and substances like quintessence, k-essence or others [5], with so far widely unknown properties and origin. The most significant and until now only known fact about such a substance is that it has to possess a negative energy density, which would be able to drive the acceleration of expansion. Mathematically this corresponds to a constant term in the Friedmann equation, similar to Einstein's cosmological constant, or an ideal fluid with negative energy density, i.e., a dependence of pressure on density as $p=w \rho$, with $w=-1$. The observed energy density is of the order of $\rho_{\Lambda, \mathrm{obs}}=10^{-47} \mathrm{GeV}^{4}$.

The standard paradigm of cosmology, known as the $\Lambda \mathrm{CDM}$ model, features several components to describe the observed universe. Besides common baryonic matter, there are two other substances present. One of them is cold dark matter (CDM), responsible for the dynamics of compact objects within galaxies and the large-scale structure formation of the universe, and the other one is a cosmological constant $\Lambda$, the cause for the expansion of the universe. With these ingredients, the equation of state parameter $w$ of the universe can be written as

$$
w=-\frac{1}{1+\Omega_{M} / \Omega_{\Lambda} a^{-3}},
$$

where $\Omega_{M}$ is the normalized density of matter (both baryonic and dark), and $\Omega_{\Lambda}$ the normalized density of dark energy in the universe. Observations [8] lead to the estimates $\Omega_{M} \sim 0.3175$ and $\Omega_{\Lambda} \sim 0.6825$, which amounts to a current value of the equation of state parameter of the total universe of $w \simeq-0.68$. Alternatively, $w$ can be obtained directly from the cosmographic analysis of supernova data [9], which leads to a value of $w=-0.7174_{-0.0964}^{+0.0922}$, close to the value predicted by the $\Lambda \mathrm{CDM}$ model. This equation of state parameter characterizes the relation between pressure and density of the net fluid in the universe and determines the kinematics of its expansion. From the continuity equation, and assuming a FLRW-universe parameterized by a scale factor $a(t)$, the density of a fluid evolves as

$$
\rho \propto a^{-3(1+w)} .
$$

An equation of state parameter $w=-1$ results in a constant energy density. Solving the first Friedmann equation with a constant energy density $\rho$ for the scale factor, this results in

$$
a(t) \propto e^{t},
$$

i.e., an exponential growth of the scale of the universe.

Besides the many attempts to model or derive dark energy, an old idea to explain dark energy is to derive it from the vacuum energy fluctuations of quantum fields [10]. We have to differentiate between bosonic and fermionic contributions to the vacuum energy, which are opposite in sign, but else have the same properties. These contributions have the desired behavior in a cosmological context, as for example a constant energy density similar to a cosmological constant, and could cause the repulsive effect which is necessary to explain the newly accelerated expansion of the universe. Unfortunately, however, the predictions for the contributions of quantum fields to the vacuum energy are divergent 
- or, depending on the method of renormalization applied, at least very large. With a momentum cutoff at Planck scale the vacuum energy amounts to a value of $\rho_{\Lambda, \mathrm{th}}=10^{76} \mathrm{GeV}^{4}$. The discrepancy between this theoretical and the observed value of the vacuum energy density is thus at least 123 orders of magnitude, representing the so-called hierarchy problem.

The existence of divergences in these expressions is notorious in QFT. Without renormalization schemes, the contributions of quantum fields to the vacuum energy are divergent, which would imply that there is an infinite energy density filling spacetime. These infinities are generally regarded as an unphysical outcome of the methods applied to renormalize the integrals, and usually the paradigm is to disregard these divergences, and only consider the energy differences which are detectable in experiments. The absolute value should be of no importance and is considered unobservable. This approach is problematic however, since there are indeed experiments in which the physical existence of the vacuum energy has been proven. The quantization of radiation waves enclosed in a metal box leads to a macroscopic force on metal plates, known as the Casimir effect, which is a direct consequence of the existence of the zero-point fluctuations of quantum fields.

On the other hand, astrophysical data tell us that the universe is expanding as if driven by a constant, but very tiny, energy density filling the complete space, in behavior much like the unphysical divergent vacuum energy contribution from quantum fields. It seems as though explaining the universe's dynamics with vacuum fluctuations of fields fails solely due to a mismatch of numbers, despite the appealing properties of the vacuum energy.

However, there are arguments to validate the identification of those vacuum fluctuations as the energy that drives the expansion, like for example by principles of symmetry. Supersymmetry [1] is a concept which predicts a correspondence between the bosonic and fermionic particle content of the universe. Due to the fact that the vacuum energies of bosons and fermions have opposite signs, this correspondence can be used to eliminate divergences in the vacuum energy by cancelation of opposite-sign terms. In maximally supersymmetric models, this balance is exact and leads to a complete elimination of all divergent contributions of bosons and fermions to the vacuum energy, whereas in other supersymmetric models divergences are canceled only partly.

However, recent experiments at LHC have produced results which are inconsistent with supersymmetry, or at least with the minimally supersymmetric models amongst them. As an example, the LHCb collaboration [12] has reported the decay of $B^{0}$ into $\mu^{+}$and $\mu^{-}$ at a very low rate, which is consistent with the standard model, but expected to be much higher in many of the simpler supersymmetric models. In general, these experiments have lead to rather discouraging results for supersymmetry [13]. Moreover, currently the universe supposedly is in the state of broken supersymmetry, which would again lead to an infinite value of the vacuum energy instead of complete cancelation of divergences.

The approach presented in this work is using a similar argument for the cancelation of divergences, but instead of invoking elaborate symmetry principles and complicated mathematical constructions to describe an abundance of new particles, it strives for finding the simplest possible balance by asking how many and which kind of particles would be needed in addition to the known particles of the standard model in order to yield a vacuum energy that is finite and can explain the accelerated expansion of the universe. The goal is to cancel the divergences among the quantum fields present in the universe by adding a small amount of new particles by hand, their number and nature dictated by some simple basic requirements to be fulfilled. By explicitly calculating the vacuum energy for particles of different types and masses, it is possible to set up a number of conditions, depending on the masses of the particles, which have to be obeyed in order to achieve the correct value of the vacuum energy. By appropriately tuning the masses of the fields, it is in principle possible to eliminate the divergences, and reduce the vacuum energy to the required tiny amount predicted by astrophysical observations.

The paper is organized as follows. In Section II we start with some general facts about the functional integral formulation of QFT for relativistic bosonic and fermionic fields and the vacuum energy of fields. Section [II presents the exact computations of the vacuum energy and sets up conditions for the cancelation of vacuum energy contributions. In Section [IV] we generalize the calculations to curved spacetimes and obtain the contributions of quantum fields to the vacuum energy in terms of an expansion of the effective action with respect to the curvature of spacetime. In Section $\nabla$ we set up the cancelation conditions in curved spacetimes, and evaluate the balance for a specific choice of spacetime in Section VI. It will be shown that it is possible, by appropriate choice of masses and fields, to fit the observed dark energy component of the universe, and that the occurring contributions of curvature in the cancelation conditions help to simplify fulfilling the conditions for the masses of the fields present. Section VII contains a conclusion.

\section{FUNCTIONAL INTEGRALS OF FREE BOSONIC AND FERMIONIC FIELDS}

\section{A. Bosonic fields}

In the simplest example of a free complex boson field $\phi$ describing charged bosons with mass $m_{\mathrm{b}}$ in flat space- 
time, we can write the relativistic Lagrangian as

$$
\mathcal{L}_{\mathrm{b}}=\eta^{\mu \nu}\left(\partial_{\mu} \bar{\phi}\right)\left(\partial_{\nu} \phi\right)-m_{\mathrm{b}}^{2} \bar{\phi} \phi
$$

where $\eta^{\mu \nu}$ is the Minkowski metric. The partition function is given by the functional integral

$$
Z_{\mathrm{b}}=\int \mathcal{D} \bar{\phi} \mathcal{D} \phi \exp \left[-i \int d^{D} x \sqrt{-\eta} \bar{\phi} G_{\mathrm{b}}^{-1} \phi\right]
$$

where $\eta=-1$ is the determinant of the Minkowski metric. With $\partial^{2}=\eta^{\mu \nu} \partial_{\mu} \partial_{\nu}$, the Greens function or propagator in position space is

$$
G_{\mathrm{b}}^{-1}=\partial^{2}+m_{\mathrm{b}}^{2}
$$

Carrying out the functional integral with the help of the Gaussian integral formula, we obtain

$$
Z_{\mathrm{b}}=\operatorname{det} G_{\mathrm{b}}=\operatorname{det}\left[\partial^{2}+m_{\mathrm{b}}^{2}\right]^{-1} \text {. }
$$

\section{B. Fermionic fields}

For a free complex massive fermion field $\psi$, the relativistic Lagrangian comes from Dirac theory,

$$
\mathcal{L}_{\mathrm{f}}=\bar{\psi}\left(i \not \partial-m_{\mathrm{f}} \mathbf{1}\right) \psi,
$$

where $\not \partial=\gamma_{i j}^{a} \partial_{a}$ is the Feynman-slashed derivative operator, and the $\gamma_{i j}^{a}$ are the Dirac $\gamma$-matrices in flat spacetime. The functional integral reads

$$
Z_{\mathrm{f}}=\int \mathcal{D} \bar{\psi} \mathcal{D} \psi \exp \left[i \int d^{D} x \sqrt{-\eta} \bar{\psi} G_{\mathrm{f}}^{-1} \psi\right] .
$$

with the inverse propagator

$$
G_{\mathrm{f}}^{-1}=i \not \partial-m_{\mathrm{f}} \mathbf{1} .
$$

Carrying out the functional integrals over $\psi$ and $\bar{\psi}$, employing a slightly different scheme than before due to the Grassmannian nature of fermion fields [14], we arrive at

$$
Z_{\mathrm{f}}=\operatorname{det} G_{\mathrm{f}}^{-1}=\operatorname{det}\left[i \not \partial-m_{\mathrm{f}} \mathbf{1}\right],
$$

Arguing that, due to the existence of the Dirac sea, the energies of Dirac fermions are symmetric around $p=0$, and considering that for an $n \times n$-matrix with even $n$, an overall sign in the determinant does not matter, we can rewrite the determinant in the partition function as

$$
Z_{\mathrm{f}}=\operatorname{det}\left[\partial^{2}+m_{\mathrm{f}}^{2} \mathbf{1}\right]^{1 / 2} .
$$

\section{Effective action}

Combining the results for the partition function for free bosons and fermions, and introducing the notion of effective action $S_{\text {eff }}$ as

$$
Z=e^{i S_{\text {eff }}}
$$

we can express the first loop contribution to the effective action for bosons as

$$
i S_{\mathrm{eff}}^{(\mathrm{b})}=\ln \operatorname{det} G_{\mathrm{b}}=-\operatorname{Tr}_{\mathrm{F}} \ln \left(\partial^{2}+m_{\mathrm{b}}^{2}\right)
$$

where $\operatorname{Tr}_{\mathrm{F}}$ denotes the trace over the functional space. In the case of fermions, we have

$$
i S_{\mathrm{eff}}^{(\mathrm{f})}=-\ln \operatorname{det} G_{\mathrm{f}}=\frac{1}{2} \operatorname{Tr}_{\mathrm{F}, \mathrm{D}} \ln \left(\partial^{2}+m_{\mathrm{f}}^{2} \mathbf{1}\right)
$$

where the subscript $D$ indicates the trace over the Dirac indices, which leads to an additional factor of four in the expression. Carrying out the Dirac trace and summarizing the results, we can write the partition function of a system with charged bosons and fermions as

$$
Z=\exp \left[-\operatorname{Tr}_{\mathrm{F}} \ln \left(\partial^{2}+m_{\mathrm{b}}^{2}\right)+2 \operatorname{Tr}_{\mathrm{F}} \ln \left(\partial^{2}+m_{\mathrm{f}}^{2}\right)\right]
$$

We see that the contributions of bosons and fermions enter with opposite sign and differ from each other by a factor of two. This mirrors the fact that complex scalar fields possess only two degrees of freedom (for charge conjugation), whereas massive Dirac fermions have four degrees of freedom (two for the charge conjugation and two for the spin orientations). Considering variations as a real scalar field, i.e., uncharged bosons, or a Majorana spinor, i.e., uncharged fermions, the partition functions would differ in the prefactor of the exponent's argument, according to the respective number of degrees of freedom. This holds for other species like vector particles as well, which have additional numerical factors due to the possible spin orientations and charges. In the example of a vector boson, its degrees of freedom can be inferred from the formulation as a superposition of plane waves with different polarization states $\epsilon^{\mu}(\nu)$. For a massive boson, three polarization modes are possible, i.e., two transverse and one longitudinal mode, whereas for a massless boson, the longitudinal mode vanishes, and thus two degrees of freedom are left. A charge signifies further multiplications of the degrees of freedom.

The general rule is that bosons and fermions yield contributions to the vacuum energy which are opposite in sign, and this fact will be used to set up a cancelation scheme. Bosons occur in the effective action with a negative sign, corresponding to a set of harmonic oscillators with positive energies, whereas fermions have negative energies and contribute positive terms to the effective action.

The one-loop contribution to the effective action, as thus formulated for bosons and fermions, is the quantity of interest to use for the balance of bosonic and fermionic contributions. Hereby we neglect any form of interaction between the particle species and consider purely free fields. To be more realistic, one would have to consider all possible further diagrams, including higher order selfinteractions, but for the purpose of demonstrating the principle of our model, we restrict ourselves to the first loop diagrams. 


\section{CALCULATING THE TOTAL VACUUM ENERGY}

In order to calculate the vacuum energy of free particles described by the Lagrangians introduced before, we transfer the problem to Fourier space, where we are left to carry out integrals of the form

$$
i S_{\mathrm{eff}} \propto \int d^{D} x \sqrt{-\eta} \int \frac{d^{D} p}{(2 \pi)^{D}} \ln \left(-p^{2}+m^{2}\right) .
$$

Integrals of that kind for the case of $D=4$ are divergent, but can be calculated using regularization techniques. A commonly used method is cutoff regularization, where the integral in phase space is carried out only up to a maximum frequency, which represents the divergence. Another option is to use dimensional regularization, which will be employed here, and which substitutes the number of spacetime dimensions $D$ by $D=d-\epsilon, d$ being an integer. This will transform the divergences into the form of poles in $\epsilon$ in the limit of physical dimensions, i.e., $\epsilon \rightarrow 0$. Whichever regularization scheme is used, there will be divergences in the resulting expression, and depending on the regularization they will take different shapes. Using dimensional regularization, for a general number of spacetime dimensions $D$, we can calculate the integral in Eq. (17]) as (see e.g. [15])

$$
\int \frac{d^{D} p}{(2 \pi)^{D}} \ln \left(-p^{2}+m^{2}\right)=-i \frac{\Gamma(-D / 2)}{(4 \pi)^{D / 2}} \frac{1}{\left(m^{2}\right)^{-D / 2}} .
$$

This integral is divergent because the $\Gamma$-function diverges for negative even integer values without any possibility of analytic continuation or similar methods. The expression can be processed further using aforementioned substitution $D=4-\epsilon$, which will make it possible to explicitly isolate the infinities in terms which diverge in the limit $\epsilon \rightarrow 0$. We use the expansion of the $\Gamma$-function for small $\epsilon$ 16],

$$
\begin{aligned}
\Gamma(-n+\epsilon) \simeq & \frac{(-1)^{n}}{n !}\left\{\frac{1}{\epsilon}+\psi(n+1)\right. \\
& \left.+\frac{\epsilon}{2}\left[\frac{\pi^{2}}{3}+\psi^{2}(n+1)-\psi^{\prime}(n+1)\right]\right\},
\end{aligned}
$$

where $\psi(n)$ is the Digamma function, and $\psi^{\prime}(n)$ the Trigamma function, and expand the mass term for small $\epsilon$ as well,

$$
m^{-\epsilon}=\mu^{-\epsilon}\left(\frac{\mu}{m}\right)^{\epsilon} \simeq \mu^{-\epsilon}\left[1+\epsilon \ln \frac{\mu}{m}+\mathcal{O}\left(\epsilon^{2}\right)\right] .
$$

Here $\mu$ is an auxiliary parameter with the dimension of a mass and of arbitrary size, introduced to make the argument of the logarithm dimensionless.

Truncating at linear order in $\epsilon$, we end up with an expression for the effective action as

$$
S_{\mathrm{eff}} \simeq-\frac{i \mu^{-\epsilon}}{(4 \pi)^{2}} m^{4}\left[\frac{1}{\epsilon}+\ln \frac{\mu}{m}+1-\frac{\gamma}{2}+\mathcal{O}(\epsilon)\right],
$$

where $\gamma$ is the Euler-Mascheroni constant. From this result, we see that in order to cancel the divergent terms proportional to $1 / \epsilon$, we need to require that the sum over all quartic powers of the masses in the system vanish,

$$
\sum_{\mathrm{b}} m_{\mathrm{b}}^{4}=\sum_{\mathrm{f}} m_{\mathrm{f}}^{4}
$$

which will thus ensure that the effective energy is finite. This will also cancel the constant terms, $1-\gamma / 2$, in the effective action. The remaining convergent terms in the effective action should subsequently be tuned to obtain the observed cosmological constant, which can be achieved by fulfilling the condition

$$
\sum_{\mathrm{b}} m_{\mathrm{b}}^{4} \ln \frac{\mu}{m_{\mathrm{b}}}-\sum_{\mathrm{f}} m_{\mathrm{f}}^{4} \ln \frac{\mu}{m_{\mathrm{f}}}=\rho_{\Lambda} .
$$

In this derivation, we have failed to obtain an additional divergence, since within the formalism of dimensional regularization quadratically divergent contributions to the integral are lost due to the application of Veltman's rule [15, 17]. We would have obtained those divergences correctly if we had used a cutoff regularization, which would have lead to an additional term in the effective action proportional to the cutoff scale, but without any mass dependence. In order to reconsider those divergences in the balance, we additionally have to require the fulfillment of another condition, i.e., the balance of the degrees of freedom $\nu_{i}$ of the particles present in the system, such that the contribution proportional to the cutoff scale will be canceled. This is achieved by the condition

$$
\sum_{\mathrm{b}} \nu_{\mathrm{b}}=\sum_{\mathrm{f}} \nu_{\mathrm{f}}
$$

With these three conditions, Eq. (22), (23) and (24), we can make the divergent contributions to the vacuum energy vanish and obtain the correct remainder to explain the accelerated expansion of the universe.

\section{RESULTS IN CURVED SPACETIME}

Before we continue to investigate how these conditions can be fulfilled, we move on to reconsider the situation in curved spacetime described by a general metric $g^{\mu \nu}$, where the occurring operators are replaced by generalized expressions in curved spacetime.

The Laplacian can be written in a conformally invariant way as the so-called generalized Laplace-Beltrami operator,

$$
\Delta_{L B}^{\mathrm{gen}}=\Delta_{L B}-\xi R=\frac{1}{\sqrt{-g}} \partial_{\mu} \sqrt{-g} g^{\mu \nu} \partial_{\nu}-\xi R .
$$

where $R$ is the curvature scalar of spacetime and $\Delta_{L B}$ is the usual Laplace operator in curved spaces. It can be shown [18] that the factor $\xi$ must be zero, on grounds of 
demanding a unique mapping between the coordinates of flat and curved spacetimes, not only for point particles, but also for extended objects of different shape like wave packets.

For fermionic fields, the Dirac $\gamma$-matrices in flat spacetime $\gamma_{i j}^{a}$ have to be replaced by

$$
\gamma_{i j}^{\mu}=e_{a}^{\mu} \gamma_{i j}^{a}
$$

where $e_{a}^{\mu}$ are the vierbein fields corresponding to the geometric description of the spacetime $g^{\mu \nu}$. Furthermore, the factor $\sqrt{-\eta}$ in the action is not equal to unity anymore, but has to be replaced by the determinant of the metric of curved spacetime, $\sqrt{-g}$.

Quantitatively the effects of curvature can be described in terms of a power series expansion, which will be introduced and discussed in detail in the next subsections.

\section{A. Curvature expansion for scalars}

The effects of curvature on the vacuum energy of fields can be calculated within the framework of a so-called Schwinger-de Witt expansion, in which the Greens function of the theory is expanded into a power series with coefficients depending on the curvature of spacetime. The theory of quantum fields in curved spacetimes in general and the calculation of Greens functions in particular have been first introduced by de Witt [19], and extensively treated in several works by Christensen, Bunch, Parker, Toms and Vassilevich [20 25]. To apply the formalism to our present purpose, we have to express the effective action, i.e., the quantitative measure for the vacuum energy of the universe, in terms of the respective Greens functions of the system.

To this end, by introducing an integral over $m^{2}$ we can rewrite the effective action for a scalar field, denoted by the superscript (0), as

$$
i S_{\mathrm{eff}}^{(0)}=-\int d^{D} x \sqrt{-g} \int d m^{2} \frac{1}{\partial^{2}+m^{2}},
$$

where we can then identify the propagator in position space as

$$
G\left(x, x^{\prime}\right)=\frac{1}{\partial^{2}+m^{2}},
$$

fulfilling the equation

$$
\left(\partial^{2}+m^{2}\right) G\left(x, x^{\prime}\right)=-\delta\left(x, x^{\prime}\right) .
$$

Following [19, 21], we define the operator acting on $G\left(x, x^{\prime}\right)$ on the left hand side as $\hat{F}$. The propagator is now written as an integral over the heat kernel $\left\langle x, s \mid x^{\prime}, 0\right\rangle$ as

$$
G\left(x, x^{\prime}\right)=-i \int_{0}^{\infty}\left\langle x, s \mid x^{\prime}, 0\right\rangle e^{-i m^{2} s} d s
$$

where $s$ is the pseudotime. According to the formalism of the Schwinger-de Witt expansion, the heat kernel can be further decomposed to

$$
\left\langle x, s \mid x^{\prime}, 0\right\rangle=\frac{i \Delta_{V M}\left(x, x^{\prime}\right)}{(4 \pi i s)^{D / 2}} e^{i \sigma / 2 s} \Omega\left(x, x^{\prime}, s\right),
$$

where $\Omega\left(x, x^{\prime}, s\right)$ is a newly introduced function to be determined, $\Delta_{V M}\left(x, x^{\prime}\right)$ is the van Vleck-Morette determinant, and $\sigma=g_{\mu \nu} \sigma^{\mu} \sigma^{\nu}$ is the geodesic difference between the points $x$ and $x^{\prime}$.

The determining equation for $\Omega\left(x, x^{\prime}, s\right)$ can be obtained by plugging the expansion for the kernel, Eq. (30), into the equation for the propagator, Eq. (28), leading to

$$
i \frac{\partial}{\partial s}\left\langle x, s \mid x^{\prime}, 0\right\rangle=\left.\hat{F}\right|_{m=0}\left\langle x, s \mid x^{\prime}, 0\right\rangle,
$$

with the operator $\hat{F}$ evaluated for $m=0$. Subsequently, the equation for the function $\Omega\left(x, x^{\prime}, s\right)$ can be found as

$$
i \frac{\partial}{\partial s} \Omega+\frac{i}{s} \Omega^{; \mu} \sigma_{; \mu}=-\Delta^{-1 / 2}\left(\Delta^{1 / 2} \Omega\right)_{; \mu}{ }^{\mu} .
$$

To solve this equation we write $\Omega$ as a power series in the pseudotime $s$,

$$
\Omega\left(x, x^{\prime}, s\right)=\sum_{j=0}^{\infty}(i s)^{j} a_{j}\left(x, x^{\prime}\right),
$$

where the $a_{j}$ are determined by the recursion relations which are obtained by plugging this ansatz back into Eq. (32):

$$
\begin{aligned}
\sigma^{; \mu} a_{0 ; \mu} & =0 \\
\sigma^{; \mu} a_{j+1 ; \mu}+(j+1) a_{j+1} & =\left.\Delta^{-1 / 2} \hat{F}\right|_{m=0}\left[\Delta^{1 / 2} a_{j}\right]
\end{aligned}
$$

with the boundary condition $a_{0}\left(x, x^{\prime}\right)=1$. This boundary condition ensures the consistency of the curvature expansion with the limit of flat spacetimes, in which all higher order coefficients with $j \geq 1$ will vanish, and only the 0 th order coefficient remains. In the coincidence limit $x \rightarrow x^{\prime}$, the van Vleck-Morette determinant becomes the unit matrix, and the geodesic distance $\sigma=g_{\mu \nu} \sigma^{\mu} \sigma^{\nu}$ between $x$ and $x^{\prime}$ becomes zero. The heat kernel then reads

$$
\langle x, s \mid x, 0\rangle=\frac{i}{(4 \pi i s)^{D / 2}} \sum_{j=0}^{\infty}(i s)^{j} a_{j}(x, x),
$$

with the first three coefficients obtained from the above recursion relations as

$$
\begin{aligned}
& a_{0}=1 \\
& a_{1}=\frac{1}{6} R, \\
& a_{2}=\frac{1}{30} \square R-\frac{1}{72} R^{2}+\frac{1}{180}\left(R_{\alpha \beta \gamma \delta} R^{\alpha \beta \gamma \delta}-R_{\alpha \beta} R^{\alpha \beta}\right) .
\end{aligned}
$$


After introducing the identity

$$
\frac{i}{(4 \pi i s)^{D / 2}} e^{-i m^{2} s}=\int \frac{d^{D} k}{(2 \pi)^{D}} e^{-i s\left(-k^{2}+m^{2}\right)},
$$

we replace the $j$ th power of pseudotime $(i s)^{j}$ with the $j$ th derivative with respect to $m^{2}$. Carrying out the integral over the pseudotime $s$ and plugging everything back into the propagator, we obtain

$$
G(x, x)=\int \frac{d^{D} k}{(2 \pi)^{D}} \sum_{j=0}^{\infty} a_{j}(x, x)\left(-\frac{\partial}{\partial m^{2}}\right)^{j}\left[\frac{1}{-k^{2}+m^{2}}\right],
$$

and thus for the effective action

$$
i S_{\mathrm{eff}}^{(0)}=-\int d^{D} x \sqrt{-g} \int d m^{2} \int \frac{d^{D} k}{(2 \pi)^{D}}\left(\frac{a_{0}}{-k^{2}+m^{2}}+\frac{a_{1}}{\left(-k^{2}+m^{2}\right)^{2}}+\frac{2 a_{2}}{\left(-k^{2}+m^{2}\right)^{3}}+\ldots\right) .
$$

We have thus obtained the effective action in terms of a series expansion in $\left(-k^{2}+m^{2}\right)^{-1}$, with coefficients depending on the curvature of spacetime. What remains to be done is carrying out the integrals over $k$ and $m^{2}$. The divergent $k$-integrals have to be calculated by a renormalization technique, similar to the divergent integrals in the case of flat spacetime treated earlier, whereas the mass integrals are straightforward. First however we will derive the curvature expansion for other species of particles, in particular spinor and vector fields.

\section{B. Curvature expansion for spinors}

The effective action for a spinor field, denoted by the superscript $(1 / 2)$, is

$$
i S_{\mathrm{eff}}^{(1 / 2)}=-\int d^{D} x \sqrt{-g} \operatorname{tr}_{\mathrm{D}} \ln (i \not \partial+m \mathbf{1})
$$

where $\operatorname{tr}_{D}$ represents the trace over the Dirac indices of the spinor field. Introducing the integral over the mass, we can rewrite the expression as

$$
i S_{\mathrm{eff}}^{(1 / 2)}=-\int d^{D} x \sqrt{-g} \int d m \operatorname{tr}_{\mathrm{D}}\left(\frac{1}{i \not \partial+m \mathbf{1}}\right),
$$

and identify the propagator in position space as

$$
G\left(x, x^{\prime}\right)=\frac{1}{i \not \partial+m \mathbf{1}},
$$

fulfilling the equation

$$
(i \not \partial+m \mathbf{1}) G\left(x, x^{\prime}\right)=-\delta\left(x, x^{\prime}\right) .
$$

However, to be able to apply the Schwinger-de Witt expansion, we need a quadratic operator, not one proportional to $\partial$ as in the case of Dirac spinors. Thus, we rewrite the effective action as outlined in Section $\amalg B$ to

$$
i S_{\mathrm{eff}}^{(1 / 2)}=\frac{1}{2} \int d^{D} x \sqrt{-g} \operatorname{tr}_{\mathrm{D}} \ln \left[\partial^{2}+m^{2} \mathbf{1}\right],
$$

now containing a quadratic operator, which is taken into account by the factor of $1 / 2$ in front of the whole expression. Introducing now the mass integral as before in the case of the scalar field, the effective action becomes

$$
i S_{\mathrm{eff}}^{(1 / 2)}=\frac{1}{2} \int d^{D} x \sqrt{-g} \int d m^{2} \operatorname{tr}_{\mathrm{D}} \frac{1}{\not^{2}+m^{2} \mathbf{1}},
$$

where now we can carry out the curvature expansion as before for the Greens function $\mathbf{G}\left(x, x^{\prime}\right)$ obeying the equation

$$
\operatorname{tr}_{\mathrm{D}}\left(\partial^{2}+m^{2} \mathbf{1}\right) \mathbf{G}\left(x, x^{\prime}\right)=-\delta\left(x, x^{\prime}\right) .
$$

Taking the trace, this equation can be brought into the form

$$
\left(\partial^{2}+\frac{1}{4} R-m^{2}\right) G\left(x, x^{\prime}\right)=-\delta\left(x, x^{\prime}\right) .
$$

The Greens function can then be written in terms of the heat kernel expansion as

$$
\mathbf{G}(x, x)=\int \frac{d^{D} k}{(2 \pi)^{D}} \sum_{j=0}^{\infty} \mathbf{a}_{j}(x, x)\left(-\frac{\partial}{\partial m^{2}}\right)^{j} \frac{1}{-k^{2}+m^{2}},
$$

with the first coefficients for spinor fields as 21]

$$
\begin{aligned}
\mathbf{a}_{0} & =\mathbf{1} \\
\mathbf{a}_{1} & =\frac{1}{12} R \mathbf{1}, \\
\mathbf{a}_{2} & =\left(\frac{1}{288} R^{2}-\frac{1}{120} \square R-\frac{1}{180} R_{\alpha \beta} R^{\alpha \beta}\right. \\
& \left.+\frac{1}{180} R_{\alpha \beta \gamma \delta} R^{\alpha \beta \gamma \delta}\right) \mathbf{1}-\frac{1}{192} \sigma_{\alpha \beta} \sigma_{\gamma \delta} R^{\alpha \beta \lambda \xi} R_{\lambda \xi}^{\gamma \delta},
\end{aligned}
$$

and $\sigma_{\alpha \beta}=\frac{i}{2}\left[\gamma_{\alpha}, \gamma_{\beta}\right]_{-}$being the commutator of the $\gamma$ matrices. Considering that in the spinor case we still have to evaluate the Dirac trace over the coefficients, the effective action is then obtained as

$$
i S_{\mathrm{eff}}^{(1 / 2)}=\frac{1}{2} \int d^{D} x \sqrt{-g} \int d m^{2} \operatorname{tr}_{\mathrm{D}} \mathbf{G}(x, x),
$$

with $\mathbf{G}(x, x)$ determined by Eqs. (48) and (49). 


\section{Curvature expansion for vector fields}

Next we investigate the expansion for a vector field included in the balance. We start with the action for a massive vector field, denoted by the superscript (1),

$$
\begin{array}{r}
S^{(1)}=\frac{1}{2} \int d^{D} x \sqrt{-g} A_{\mu}\left[g^{\mu \nu}\left(\partial^{2}+m^{2}\right)\right. \\
\left.-\left(1-\frac{1}{\alpha}\right) \partial^{\mu} \partial^{\nu}\right] A_{\nu} .
\end{array}
$$

We include a gauge-fixing term to account also for the possibility of taking the limit $m \rightarrow 0$ later, which considers the case of photons or gluons. The operator in the action is identified as the inverse of the Greens function,

$$
\left(G^{\mu \nu}\right)^{-1}\left(x, x^{\prime}\right)=g^{\mu \nu}\left(\partial^{2}+m^{2}\right)-\left(1-\frac{1}{\alpha}\right) \partial^{\mu} \partial^{\nu},
$$

and obeys the equation

$$
\left[g^{\mu \nu}\left(\partial^{2}+m^{2}\right)-\left(1-\frac{1}{\alpha}\right) \partial^{\mu} \partial^{\nu}\right] G_{\mu \nu}\left(x, x^{\prime}\right)=-\delta\left(x, x^{\prime}\right) .
$$

The effective action then reads

$$
i S_{\mathrm{eff}}^{(1)}=-\int d^{D} x \sqrt{-g} \operatorname{tr}_{\mathrm{L}} \ln \left(G^{\mu \nu}\right)^{-1}\left(x, x^{\prime}\right),
$$

where $\operatorname{tr}_{\mathrm{L}}$ denotes the trace over the Lorentz indices. The introduction of a mass integral can be done considering the rules of matrix computation and logarithms, and leads to

$$
i S_{\mathrm{eff}}^{(1)}=-\int d^{D} x \sqrt{-g} \int d m^{2} \operatorname{tr}_{\mathrm{L}}\left[g^{\mu \lambda} G_{\lambda \nu}\left(x, x^{\prime}\right)\right] .
$$

The matrix $G_{\lambda \nu}\left(x, x^{\prime}\right)$ can be expanded and calculated in the same way as the propagators before in the framework of a Schwinger-de Witt expansion. The case of vector fields has been extensively treated in [21], where the first three coefficients in the coincidence limit were found to be

$$
\begin{aligned}
a_{0 \lambda \nu}= & \delta_{\lambda \nu} \\
a_{1 \lambda \nu}= & \frac{1}{6}\left(R g_{\lambda \nu}-R_{\lambda \nu}\right), \\
a_{2 \lambda \nu}= & {\left[-\frac{1}{6} R R_{\lambda \nu}-\frac{1}{6} \square R_{\lambda \nu}+\frac{1}{2} R_{\lambda \alpha} R_{\nu}^{\alpha}-\frac{1}{12} R_{\nu}^{\alpha \beta \gamma} R_{\alpha \beta \gamma \lambda}\right.} \\
& \left.\quad+\left(\frac{1}{72} R^{2}+\frac{1}{30} \square R-\frac{1}{180} R_{\alpha \beta} R^{\alpha \beta}+\frac{1}{180} R_{\alpha \beta \gamma \delta} R^{\alpha \beta \gamma \delta}\right) g_{\lambda \nu}\right] .
\end{aligned}
$$

Note that these results have been obtained using the Feynman gauge, i.e., $\alpha=1$. Within the effective action, we then have to consider an additional contraction with the metric, i.e., calculate the expressions $g^{\mu \lambda} a_{j \lambda \nu}$, and then take the trace over the Lorentz indices $\mu, \nu$.

\section{BALANCING THE EFFECTIVE ACTION}

To obtain the final expressions for the effective actions, we investigate the obtained expressions limiting the calculations to the first three terms in the expansion of the Greens function, i.e., $j \leq 2$. We have to solve integrals of the form

$$
I_{\alpha}(D)=\int \frac{d^{D} k}{(2 \pi)^{D}} \frac{1}{\left(-k^{2}+m^{2}\right)^{\alpha}}
$$

for $\alpha=1,2,3$. Similarly to before, the results can be expressed in terms of the Gamma function,

$$
I_{\alpha}(D)=\frac{i}{(4 \pi)^{D / 2}} \frac{\Gamma\left(\alpha-\frac{D}{2}\right)}{\Gamma(\alpha)} \frac{1}{\left(m^{2}\right)^{\alpha-D / 2}} .
$$

We use dimensional regularization to further process those integrals, setting $D=4-\epsilon$, with $\epsilon$ taken to zero in the end. In further calculations, 'safe' limits $\epsilon \rightarrow 0$ will be taken immediately. The integral with $\alpha=3$ is convergent and can be expressed directly, whereas the cases $\alpha=1,2$ contain divergences which have to be investigated:

$$
\begin{aligned}
& I_{1}(4-\epsilon)=\frac{i}{(4 \pi)^{2}} \Gamma\left(\frac{\epsilon}{2}-1\right)\left(m^{2}\right)^{1-\epsilon / 2} \\
& I_{2}(4-\epsilon)=\frac{i}{(4 \pi)^{2}} \Gamma\left(\frac{\epsilon}{2}\right)\left(m^{2}\right)^{-\epsilon / 2} \\
& I_{3}(4-\epsilon)=\frac{i}{2(4 \pi)^{2}} \frac{1}{m^{2}} .
\end{aligned}
$$


Before considering the divergences however, we carry out the mass integrations, which in the case of the scalar field leads to an effective action of

$$
S_{\mathrm{eff}}^{(0)}=-\frac{1}{8 \pi^{2}} \int d^{4-\epsilon} x \sqrt{-g}\left[a_{0} \Gamma\left(\frac{\epsilon}{2}-1\right) \frac{m^{4-\epsilon}}{4-\epsilon}+a_{1} \Gamma\left(\frac{\epsilon}{2}\right) \frac{m^{2-\epsilon}}{2-\epsilon}+a_{2} \ln m-\ldots\right],
$$

with the coefficients $a_{j}$ given by Eqs. (36). For the spinor field, denoted by the superscript (1/2), integrating out the mass is again straightforward. After taking the Dirac trace, we can write the effective action for spinor fields in the same form as for scalars, but with the opposite sign, and with the new coefficients $\tilde{a}_{i}$,

$$
\begin{aligned}
\tilde{a}_{0} & =2 \\
\tilde{a}_{1} & =\frac{1}{6} R \\
\tilde{a}_{2} & =\frac{1}{144} R^{2}-\frac{1}{60} \square R-\frac{1}{90} R_{\alpha \beta} R^{\alpha \beta}+\frac{1}{90} R_{\alpha \beta \gamma \delta} R^{\alpha \beta \gamma \delta} \\
& +\frac{1}{96} \operatorname{tr}_{\mathrm{D}}\left[\sigma_{\alpha \beta} \sigma_{\gamma \delta} R_{\lambda \beta}^{\alpha \beta \xi} R_{\lambda \xi}^{\gamma \delta}\right] .
\end{aligned}
$$

In the third case of the vector bosons, the effective action is basically the same as in the scalar boson case, including the sign, but with coefficients where the trace over the Lorentz indices still has to be taken.

As noted earlier, the effective action for bosons is negative, corresponding to positive physical energies, whereas for fermions the effective action is positive, implying negative energies. In order to obtain a reasonable result in the limit $\epsilon \rightarrow 0$, we can now expand the Gamma functions as in (19), and the mass term as in (20). Plugging these expressions into the effective actions, and omitting all terms of $\mathcal{O}(\epsilon)$, we end up with

$$
S_{\text {eff }}=-\frac{1}{8 \pi^{2}} \int d^{4} x \sqrt{-g}\left[\mathfrak{a}_{0} \frac{m^{4}}{4}\left(\frac{2}{\epsilon}+\frac{1}{2}-\gamma-2 \ln \frac{\mu}{m}\right)+\mathfrak{a}_{1} \frac{m^{2}}{2}\left(\frac{2}{\epsilon}-\gamma-2 \ln \frac{\mu}{m}\right)+\mathfrak{a}_{2} \ln m+\ldots\right]
$$

where

$$
\mathfrak{a}_{j}= \begin{cases}a_{j} & \text { scalar fields } \\ -\tilde{a}_{j} & \text { spinor fields } \\ \operatorname{tr}_{\mathrm{L}}\left[g^{\mu \lambda} a_{j \lambda \nu}\right] & \text { vector fields }\end{cases}
$$

In expression (64), considering $\epsilon \rightarrow 0$, we have now clearly isolated the divergent and convergent parts of the effective action, and can proceed to the argument of cancelation. The aim is to obtain an effective action which does not contain any divergent terms and whose convergent remainder is small enough to explain the perceived accelerated expansion of the universe. In order to eliminate the divergent contributions to the effective action, the masses $m_{i}$ of the particles in the system have to fulfill the condition

$$
\sum_{i}\left[\mathfrak{a}_{0, i} \frac{m_{i}^{4}}{4}+\mathfrak{a}_{1, i} \frac{m_{i}^{2}}{2}\right]=0
$$

Then the remaining convergent part of the effective action must have exactly the size of the observed cosmological constant driving the accelerated expansion, i.e., the system of masses must simultaneously obey

$$
\begin{aligned}
& \frac{1}{8 \pi^{2}} \sum_{i}\left[-\mathfrak{a}_{0, i} \frac{m_{i}^{4}}{2} \ln m_{i}\right. \\
& \left.\quad+\mathfrak{a}_{1, i} m_{i}^{2}\left(-\frac{1}{4}-\ln m_{i}\right)+\mathfrak{a}_{2, i} \ln m_{i}\right]=\rho_{\Lambda} .
\end{aligned}
$$

These two conditions can, for a system with any number of particles, be fulfilled by the introduction of two new masses. The factor of $\mu$ in the log-terms has not been included here since these terms can be eliminated by condition (66) - simply by separating the log of the fraction into a sum of two logs, the $\ln \mu$-term then represents a constant factor which is canceled by the balance of divergences. As a consequence, the terms in Eq. (67), in particular the terms proportional to $\mathfrak{a}_{0}$ and $\mathfrak{a}_{1}$, changed sign, and thus the contributions of bosons to the subleading convergent terms of the effective action are now positive, and those of fermions are negative.

We would like to return shortly to the case of massless particles, in particular massless vector bosons like the photon or gluons. From the effective action for vector bosons by analogy with Eq. (39), for massless particles in 
the limit $m \rightarrow 0$ we have to solve integrals of the form

$$
I_{\alpha}(D)=\int \frac{d^{D} k}{(2 \pi)^{D}} \frac{1}{\left(k^{2}\right)^{\alpha}} .
$$

However, these integrals are zero for $D, \alpha \in \mathbb{C}$ when using the formalism of dimensional regularization, due to Veltman's formula [15, 17]. That naïvely implies that the contributions of massless vector bosons, or massless particles in general, are zero. However, we know that there are the kinetic energy contributions to the effective action even from massless particles, as argued before in Section [II] which are lost due to the use of dimensional regularization. Thus, to compensate for the missing divergences, we recall another condition to be fulfilled, namely the balance of degrees of freedom, as already stated in (24):

$$
\sum_{\mathrm{b}} \nu_{\mathrm{b}}=\sum_{\mathrm{f}} \nu_{\mathrm{f}}
$$

In this balance of degrees of freedom, as well as in condition (66), bosons as usual give negative contributions to the effective action, and fermions enter with positive signs. Only in the sub-leading convergent remainder, the signs change and bosons occur with positive and fermions with negative contributions.

In summary, we have three conditions to be fulfilled by a system of particles in order to reproduce the observed effect of accelerated expansion, i.e., Eqs. (66), (67) and (69). We shall see that it is possible to fulfill these three conditions by the introduction of only two new particles to the system.

\section{DARK ENERGY FROM THE CURVATURE TERMS IN THE EFFECTIVE ACTION}

In the previous sections, we have obtained expressions for the vacuum energy of a system of bosons and fermions as an expansion in terms of the curvature of the system, and set up conditions for the cancelation of the effective action depending on the particle masses and the curvature of spacetime. For a FLRW-universe described by the metric

$$
d s^{2}=d t^{2}-a(t)^{2}\left(d x^{2}+d y^{2}+d z^{2}\right)
$$

we can now calculate the relevant curvature quantities like the curvature scalar, the Ricci tensor and the Riemann tensor, and then proceed to compute the coefficients of the heat kernel expansion as given by Eqs. (36), (56), and (63). The results can be found in the appendix. They are expressions depending on the scale factor and its derivatives, which can be recast as a function of a series of cosmological parameters, the socalled cosmographic series (CS). The most prominent of them is the Hubble parameter $H_{0}$, followed by several more, defined as

$$
\begin{aligned}
H & \equiv \frac{1}{a} \frac{d a}{d t}, & q \equiv-\frac{1}{a H^{2}} \frac{d^{2} a}{d t^{2}}, \\
j & \equiv \frac{1}{a H^{3}} \frac{d^{3} a}{d t^{3}}, & s \equiv \frac{1}{a H^{4}} \frac{d^{4} a}{d t^{4}} .
\end{aligned}
$$

The parameter $q$ is dubbed acceleration parameter, since it describes the acceleration behavior of the universe. Further we have the jerk parameter $j$, capturing inflection points in the kinematic history of the universe, and the snap parameter $s$ from the next order of expansion. From the analysis of experimental data, in particular the numerical fits of the luminosity-redshift relation of supernovae events, it is possible to obtain numerical values for these parameters at the current time $t_{0}$. In [9] the best fit for the CS assuming the validity of the $\Lambda$ CDM model was obtained as

$$
\begin{aligned}
H_{0} & =74.05, & & q_{0}=-0.663, \\
j_{0} & =1, & & s_{0}=-0.206 .
\end{aligned}
$$

where $H_{0}$ is given in units of $\mathrm{km} / \mathrm{s} / \mathrm{Mpc}$, and the parameters $q_{0}, j_{0}$ and $s_{0}$ are dimensionless. We can thus express the coefficients of the curvature expansion in terms of these parameters, with the results to be found in the appendix as well.

If we assume the vacuum energy to be the cause of the accelerated expansion of the universe, corresponding to a cosmological constant like the one introduced by Einstein into his field equations, it occurs in the $\Lambda \mathrm{CDM}$ action as

$$
S_{\Lambda \mathrm{CDM}}=\int d^{D} x \sqrt{-g}\left[\frac{1}{2 \kappa}(R-2 \Lambda)+\mathcal{L}_{M}\right],
$$

with $\kappa=8 \pi G$, and $\mathcal{L}_{M}$ representing all matter contributions. The energy density ascribed to the cosmological constant is then $\rho_{\Lambda}$, defined as

$$
\rho_{\Lambda}=\Lambda / \kappa .
$$

From observations, the magnitude of this energy density can be inferred [7] to be

$$
\rho_{\Lambda} \simeq 10^{-122} \rho_{P} \simeq 10^{-47} \mathrm{GeV}^{4},
$$

where $\rho_{P} \equiv m_{P}^{4}$ is the Planck density, and we use a unit system with $c=\hbar=1$. This energy density has to be reproduced from the contributions of bosons and fermions in the system. The cosmological term enters into the action with a negative sign. Thus, since according to Eq. (67) fermions give a negative contribution to the convergent remainder of the effective action, we need ultimately a tiny fermionic excess in the vacuum energy. Considering all of the above input, i.e., the cancelation of the degrees of freedom, and the balancing conditions in order to achieve cancelation of divergences and make the convergent part equal to the observed cosmological constant, using the curvature coefficients calculated for 


\begin{tabular}{c|c|c|c}
\hline \hline Spin & name & mass & deg. of freed. \\
\hline \hline 0 & $H$ & $125.3 \mathrm{GeV}$ & 1 \\
\hline $1 / 2$ & $u, \bar{u}$ & $2.4 \mathrm{MeV}$ & 4 \\
& $d, \bar{d}$ & $4.8 \mathrm{MeV}$ & 4 \\
& $c, \bar{c}$ & $1.27 \mathrm{GeV}$ & 4 \\
& $s, \bar{s}$ & $104 \mathrm{MeV}$ & 4 \\
& $t, \bar{t}$ & $171.2 \mathrm{GeV}$ & 4 \\
& $b, \bar{b}$ & $4.2 \mathrm{GeV}$ & 4 \\
& $e^{-}, e^{+}$ & $0.511 \mathrm{MeV}$ & 4 \\
& $\mu^{-}, \mu^{+}$ & $105.7 \mathrm{MeV}$ & 4 \\
& $\tau^{-}, \tau^{+}$ & $1.777 \mathrm{GeV}$ & 4 \\
& $\nu_{e}, \overline{\nu_{e}}$ & $<2.2 \mathrm{eV}$ & 2 \\
& $\nu_{\mu}, \overline{\nu_{\mu}}$ & $<0.17 \mathrm{MeV}$ & 2 \\
& $\nu_{\tau}, \overline{\nu_{\tau}}$ & $<15.5 \mathrm{MeV}$ & 2 \\
\hline 1 & $\gamma$ & 0 & 2 \\
& $8 g$ & 0 & $8 \cdot 2$ \\
& $Z^{0}$ & $91.2 \mathrm{GeV}$ & 3 \\
& $W^{+}, W^{-}$ & $80.4 \mathrm{GeV}$ & $2 \cdot 3$ \\
\hline \hline
\end{tabular}

TABLE I: Particles of the standard model.

the case of a FLRW universe, we can now consider a system of particles and investigate how the aim of explaining the accelerated expansion of the universe can be accomplished. For the particle content, we use the standard model of particle physics, containing one scalar boson, nine Dirac fermions, three Majorana fermions and three vector bosons in the massive sector, and further the photon and eight gluons as massless vector bosons, as shown in Tab【. The resulting effective action is dominated by fermionic contributions, so in order to balance the sums and achieve $\rho_{\Lambda}$, new bosonic particles are needed to compensate the fermionic divergences as well as to reduce the fermionic excess in the convergent remainder down to the small value of the observed vacuum energy. It turns out that considering the masses and the curvature coefficients in the chosen unit system of $\mathrm{GeV}^{4}$ with $c=\hbar=1$, in the balance (67) the third term becomes negligible since its order of magnitude lies far below that of $\rho_{\Lambda}$. We can thus simplify (67) to

$\frac{1}{8 \pi^{2}} \sum_{i}\left[-\mathfrak{a}_{0, i} \frac{m_{i}^{4}}{2} \ln m_{i}+\mathfrak{a}_{1, i} m_{i}^{2}\left(-\frac{1}{4}-\ln m_{i}\right)\right]=\rho_{\Lambda}$.

Since we have two mass balance equations to be fulfilled, i.e., Eqs. (66) and (76), we need to postulate at least two new bosons. Further, we need to fulfill the balance of the degrees of freedom. Due to the abundance of fermions, $42-28=14$ additional bosonic degrees of freedom are required to fulfill Eq. (69). This gives constraints on the nature of the new bosons, i.e., on their spin and charge. The charges involved are probably none of those known from the standard model, since in that case the new particles should participate in the interactions with the stan- dard model particles and be detected in experiments. In the case of two new particles, the 14 degrees of freedom can be distributed in a $4+10$ manner, i.e., ten of the 14 degrees of freedom could be due to a spin 2 particle with a $U(1)$ type of charge, while the four remaining degrees of freedom could be explained by a spin 1 particle with a more complicated construction of charges, possibly similar to the $U(1) \times S U(2)$ group of the electroweak theory. Thus postulating two new particles, one vector and one tensor boson, from Eqs. (66) and (76) we can calculate their masses as

$$
\begin{aligned}
m_{V} & =168.9 \mathrm{GeV}, \\
m_{T} & =67.7 \mathrm{GeV} .
\end{aligned}
$$

These two new particles with the above mentioned properties can thus reproduce the desired value of the vacuum energy corresponding to the acceleration behavior of the universe. The massive tensor boson is reminiscent of the as yet unknown quantized form of the gravitational field. Of course, this is only the minimal solution of our conditions in order to achieve cancelation. In principle, it is possible to fulfill the conditions with combinations of more than two particles - we are free to play with the balance of the degrees of freedom and the masses of the newly introduced particles. Instead of the above construction, the degrees of freedom could also be distributed to three new particles in a $3+10+1$ manner, with an uncharged vector boson with mass $m_{V}$, a charged tensor boson with mass $m_{T}$ and a massless uncharged scalar field $S$ accounting for the last degree of freedom. Another possibility would be to consider three massive particles in the balance. However, then the system of equations is overdetermined, and one of the three particles can be chosen arbitrarily. In one variation of a $3+10+1$ distribution of degrees of freedom, one could consider the axion field [26] as the uncharged scalar boson, with its mass generally assumed to be bound by the order of $m_{a} \lesssim 1 \mathrm{eV}$, and, as before, two higher spin fields like one uncharged vector and one charged tensor. The masses of the vector and tensor bosons change due to the introduction of the axion field, and are calculated as

$$
\begin{aligned}
m_{V} & =57.6 \mathrm{GeV}, \\
m_{T} & =174.3 \mathrm{GeV} .
\end{aligned}
$$

As a last example, we considered a $2+6+6$ distribution of degrees of freedom, assuming a charged scalar field and two charged vector fields. Choosing the mass of the charged scalar boson to be again of the order of the axion mass, we determined the masses of the two vector bosons as

$$
\begin{aligned}
& m_{V 1}=76.9 \mathrm{GeV}, \\
& m_{V 2}=117.3 \mathrm{GeV} .
\end{aligned}
$$

The different examples proposed here are summarized in Tab[I] where the respective type, mass and degrees of freedom are listed. 


\begin{tabular}{c|c|c|c}
\hline \hline Spin & name & mass & deg. of freed. \\
\hline \hline 1 & $V$ & $168.9 \mathrm{GeV}$ & 4 \\
\hline 2 & $T$ & $67.7 \mathrm{GeV}$ & 10 \\
\hline \hline 0 & $S$ & 0 & 1 \\
\hline 1 & $V$ & $168.9 \mathrm{GeV}$ & 3 \\
\hline 2 & $T$ & $67.7 \mathrm{GeV}$ & 10 \\
\hline \hline 0 & $a$ & $1 \mathrm{eV}$ & 1 \\
\hline 1 & $V$ & $174.3 \mathrm{GeV}$ & 3 \\
\hline 2 & $T$ & $57.6 \mathrm{GeV}$ & 10 \\
\hline \hline 0 & $a^{+}, a^{-}$ & $1 \mathrm{eV}$ & 2 \\
\hline 1 & $V_{1}$ & $76.8 \mathrm{GeV}$ & $2 \cdot 3$ \\
& $V_{2}$ & $117.3 \mathrm{GeV}$ & $2 \cdot 3$ \\
\hline \hline
\end{tabular}

TABLE II: Proposed extensions of the standard model.

Note that in order to achieve the balances, the masses of the particles need to be fine-tuned to a very high degree, since the leading terms in the balancing condition are many orders of magnitude higher than zero, or the size of the energy density $\rho_{\Lambda}$ that we would like to achieve, respectively. The heaviest particles of the standard model, i.e., the Higgs, the top quark and the $W^{ \pm}$ bosons, dictate the order of magnitude of the masses of the new particles, since they give the most prominent contributions to the vacuum energy to be balanced. Thus, in any combination of new fields postulated, at least two heavy fields are to be expected.

\section{CONCLUSIONS}

In this paper, we have addressed the phenomenon of the accelerated re-expansion of the universe, i.e., the dark energy conundrum, or, equivalently, the hierarchy problem, dealing with the unphysical infinities contributing to the vacuum energy of quantum fields that arise from quantum field theory.

Usually argued away by renormalization procedures, we have acknowledged their physical reality, and succeeded in finding a model in which their very existence is necessary and useful to explain the present expansion rate of the universe. The zero-point fluctuations of quantum fields have all the desired properties to be an adequate candidate for dark energy, i.e., the spatially constant energy density and the repulsive effect driving the acceleration of the universe. However, the vacuum energy itself is an infinite or very large quantity, which is usually treated with diverse methods of renormalization, and seems unfit to be used as the origin of a physical phenomenon. Some theories like supersymmetry have tried to approach the problem in a different way, arguing that the vacuum energy contributions could result in a finite quantity by means of a balance of contributions, taking into account that the zero point energy has different signs for bosonic and fermionic species. In this article, we recycle the main idea of supersymmetry, i.e., the balance of the vacuum energy by boson and fermion contributions, but restrict ourselves to a much more inornate framework. We computed the vacuum energy for different species within the formalism of conventional quantum field theory, and tried to obtain a balance of contributions by postulating the existence of new particles, employing the mutual cancelation of bosons and fermions in the vacuum energy. We carried out our calculations in flat and curved spacetimes, for the latter case using a formalism known as heat kernel expansion of the Greens function, i.e., the expansion of the propagator in terms of a power series, with coefficients proportional to the curvature of spacetime. It turns out that in order to achieve the correct expansion behavior of the universe today, it is necessary to postulate the existence of at least two new bosonic particles with appropriate properties to fulfill the required 14 degrees of freedom. Several possible examples of standard model extensions have been calculated, resulting in all cases in at least two heavy fields with masses of the order of $10-10^{2} \mathrm{GeV}$. Adding those particles to the standard model, the vacuum energy obtained from conventional quantum field theories can be tuned exactly to the value of the energy density of expansion determined by astrophysical observations.

Of course, this solution to the dark energy problem is achieved by fine-tuning - the masses of the additional particles are required to be determined highly accurately in order to exactly cancel the infinities and result in the correct value of the finite remainder of the vacuum energy. However, it is remarkable that already with the introduction of only two new particles, it is in principle possible to give meaning to a seemingly nonsensical prediction of modern quantum theories, and at the same time resolve an as yet unexplained phenomenon in astrophysics. Moreover, we emphasize that the newly introduced particles are of generic nature and have no different properties than other fields of the same type and spin known so far, unlike in most other theories of dark energy, where new kinds of fields are introduced with very different and exotic properties than the known types of matter.

Finally, we would like to remark that despite its success, the model presented in this work is not completely realistic, since it assumes only non-interacting free particles, whereas we know that in reality there is an abundance of interactions between particles. These interactions of course contribute to the vacuum energy, which means that they alter the balance, and thus the result which we obtained. Furthermore, even though considering $\Lambda \mathrm{CDM}$ as the basis of our work, we have not included the cold dark matter component into the standard model particle content of our calculations, due to the lack of consolidated knowledge on this particle species. We have however considered the axion, or an axion-like 
particle, in two of our examples. In principle, some of the heavy fields postulated in our investigations could well be dark matter candidates as well. In this context, our calculations are an indication for the existence of further WIMP-like bosonic dark matter fields.

Finally it should be mentioned that a cosmological constant is needed for explaining the re-acceleration of the universe only if one assumes the cosmos to be completely isotropic and homogeneous. However, simulations of the cosmological evolution taking into account the dominance of dark matter in the gravitational attraction of the galaxies [27, 28] have shown that matter has a fractal distribution in space. Thus there is a strong influence of inhomogeneities upon the equation of motion of the size parameter of the Universe, which could result in an effect just like the one of a cosmological constant [29], i.e., a constant term in the Friedmann equation. Assuming a non-Gaussian nature of these fluctuations, and using a coordinate space cutoff at the radius of the universe to obtain a finite value of the energy, one may expect that the resulting value is indeed of the right magnitude [30]. Thus, depending on whether the assumption of homogeneity and isotropy of the universe actually holds true, the approaches to ex- plain the acceleration of expansion differ fundamentally. In the case of a homogeneous and isotropic universe however, we have demonstrated that balancing the infinite vacuum energy to result in a finite value is in principle possible by the introduction of only a small number of new particles. This should be regarded as the main conclusion here. Further efforts could be dedicated to more detailed calculations of the vacuum energy considering the various interactions between the particles of the standard model, and the more speculative sector of the particle content of the universe.

\section{Acknowledgments}

We would like to thank the anonymous referee for useful suggestions for a better illustration of our model. The work of C.G. was partly supported by the Erasmus Mundus Joint Doctorate Program by Grant Number 2010-1816 from the EACEA of the European Commission.
[1] A. G. Riess et al., Astronom. J. 116, 1009 (1998); S. Perlmutter et al., Astrophys. J. 517, 565 (1999);

[2] T. Padmanabhan, Gen. Rel. Grav. 40, 529 (2008).

[3] T. P. Sotiriou, V. Faraoni, Rev. Mod. Phys. 82, 451 (2010).

[4] R. Durrer, R. Maartens, Gen. Rel. Grav. 40, 301 (2008).

[5] C. Wetterich, Nucl. Phys. B, 302, 4, 668 (1988).

[6] P. J. E. Peebles, B. Ratra, Rev. Mod. Phys. 75, 559 (2003).

[7] E. J. Copeland, M. Sami and S. Tsujikawa, Int. J. Mod. Phys. D 15, 1753 (2006).

[8] P. A. R. Ade et al. (Planck Collaboration), Astron. \& Astrophys. preprint, arXiv:astro-ph/1303.5076 (2013).

[9] A. Aviles et al., Phys. Rev. D 86, 123516 (2012).

[10] A. D. Sakharov, Gen. Rel. Grav. 32, 2 (2000).

[11] S. P. Martin, arXiv:hep-ph/9709356 (1997).

[12] R. Aaij et al. (LHCb Collaboration), Phys. Rev. Lett. 110, 021801 (2013).

[13] M. Shifman, arXiv:physics.pop-ph/1211.0004 (2012).

[14] H. Kleinert, Path Integrals in Quantum Mechanics, Statistics, Polymer Physics, and Financial Markets, World Scientific, Singapore, 2009 (http://klnrt.de/b5).

[15] H. Kleinert, V. Schulte-Frohlinde, Critical Properties of $\phi^{4}$ theories, World Scientific, Singapore (2001): Chapter
8, p. 107, Eq. (8.27) (http://klnrt.de/b8)

[16] See Ref. [15], Chapter 8, p. 128, Eq. (8.24).

[17] G. Leibbrandt, Rev. Mod. Phys. 74, 849 (1975).

[18] See Ref. [14], Chapter 10.3.2, Chapter 11.5.

[19] B. de Witt, Dynamical Theory of Groups and Fields, Gordon and Breach, New York (1965).

[20] S. M. Christensen, Phys. Rev. D 14, 2490 (1976).

[21] S. M. Christensen, Phys. Rev. D 17, 946 (1978).

[22] T. S. Bunch, L. Parker, Phys. Rev. D 20, 2499 (1979).

[23] L. Parker, D. J. Toms, Phys. Rev. D (Rapid Communications) 31 4, 953 (1985).

[24] L. Parker, D. J. Toms, Phys. Rev. D 31 10, 2424 (1985).

[25] D. V. Vassilevich, Phys. Rep. 388, 5-6, 279 (2003).

[26] R. D. Peccei, H. R. Quinn, Phys. Rev. Lett. 38 (25), 1440 (1977).

[27] V. Springel et al., Nature 435, 629 (2005).

[28] J. Einasto et al., Astron. \& Astrophys. 534, A128 (2011).

[29] T. Buchert, M. Kerscher, C. Sicka, Phys. Rev. D 62, 043525 (2000); M. Kerscher, T. Buchert, T. Futamase, Astrophys. J. 558, L79 (2001); T. Buchert, Gen. Rel. Grav. 40, 467 (2008).

[30] H. Kleinert, http://klnrt.de/talks/CastTaiw.pptx; http://klnrt.de/403 (2012); http://klnrt.de/406 (2013); http://klnrt.de//399/399-TAIPEH.pdf (2012). 


\section{Appendix A: Coefficients for FLRW universe}

These are the coefficients of the Greens function expansions for scalar, spinor and vector particles, calculated in the case of a FLRW universe.

$$
\begin{aligned}
& a_{0}=\frac{\tilde{a}_{0}}{2}=1 \\
& a_{1}=\tilde{a}_{1}=\frac{\left[a^{\prime}(t)^{2}+a(t) a^{\prime \prime}(t)\right]}{a(t)^{2}}, \\
& a_{2}=\frac{51 a^{\prime}(t)^{4}-20 a(t) a^{\prime}(t)^{2} a^{\prime \prime}(t)+21 a(t)^{2} a^{\prime \prime}(t)^{2}+6 a(t)^{3} a^{(4)}(t)}{30 a(t)^{4}}, \\
& \tilde{a}_{2}=\frac{\left[-37+5 a(t)^{2}\right] a^{\prime}(t)^{4}+140 a(t) a^{\prime}(t)^{2} a^{\prime \prime}(t)+6 a(t)^{2}\left[3 a^{\prime \prime}(t)^{2}-2 a(t) a^{(4)}(t)\right]}{240 a(t)^{4}}, \\
& \operatorname{tr}_{\mathrm{L}}\left[g^{\mu \lambda} a_{0 \lambda \nu}\right]=4 \text {, } \\
& \operatorname{tr}_{\mathrm{L}}\left[g^{\mu \lambda} a_{1 \lambda \nu}\right]=\frac{-\left[1+3 a(t)^{2}\right] a^{\prime}(t)^{2}+2 a(t) a^{\prime \prime}(t)}{a(t)^{2}}, \\
& \operatorname{tr}_{\mathrm{L}}\left[g^{\mu \lambda} a_{2 \lambda \nu}\right]=\frac{3 a^{\prime}(t)^{4}\left[-17+41 a(t)^{2}\right]+10 a(t) a^{\prime}(t)^{2} a^{\prime \prime}(t)\left[14-15 a(t)^{2}\right]}{30 a(t)^{4}} \\
& +\frac{a(t)^{2}\left\{-2 a^{\prime \prime}(t)^{2}\left[11+12 a(t)^{2}\right]+a(t) a^{(4)}(t)\left[3+a(t)^{2}\right]\right\}}{10 a(t)^{4}} \\
& -a(t)^{2} a^{\prime}(t) a^{(3)}(t)\left[1+3 a(t)^{2}\right] .
\end{aligned}
$$

Using the definitions of the parameters of the cosmographic series, we can re-express the curvature coefficients in terms of the CS as

$$
\begin{aligned}
a_{0} & =\tilde{a}_{0}=1 \\
a_{1} & =\tilde{a}_{1}=-H_{0}^{2}\left(+1-q_{0}\right) \\
a_{2} & =\frac{H_{0}^{4}}{30}\left(51+20 q_{0}+21 q_{0}^{2}+6 s_{0}\right) \\
\tilde{a}_{2} & =\frac{H_{0}^{4}}{120}\left(-16-70 q_{0}+9 q_{0}^{2}-6 s_{0}\right) \\
\operatorname{tr}_{\mathrm{L}}\left[g^{\mu \lambda} a_{0 \lambda \nu}\right] & =4 \\
\operatorname{tr}_{\mathrm{L}}\left[g^{\mu \lambda} a_{1 \lambda \nu}\right] & =-2 H_{0}^{2}\left(2+q_{0}\right) \\
\operatorname{tr}_{\mathrm{L}}\left[g^{\mu \lambda} a_{2 \lambda \nu}\right] & =\frac{H_{0}^{4}}{15}\left(36+5 q_{0}-69 q_{0}^{2}-60 j_{0}+6 s_{0}\right) .
\end{aligned}
$$

